


\section{STILKES RECHTSBIBLIOTHEK}

Die Gesetze des Deutschen Reichs mit systematischen Erläuterungen.

\section{Bürgerliches und Handelsrecht.}

A uert, Hermann. Die Eheauflossung im neuen deutschen Recht unter Berăcksichtigung der neuesten Bestimmungen. (N a r n berger Gese tze.) 159 Seiten. Preis in Ganzleinen geb. 4,60 RM.

D a h m, A u g s t. Kleinwohnungs- und Siedlungsrecht. 160 Seiten. Preis in Ganzleinen ged. 5,50 RM.

D a h m, A ugus t. Mieterschntzgesetz. Mit Nachtrag. 162 und 4 Seiten. Preis in Ganzleinen geb. 5,- RM.

Dah m, A u g ust. Gesetzliche Miete. Reichsmietengesetz in der Fassung der Bekanntgabe vom 24. 4.1936. Etwa 100 Seiten. Pleis kart. etwa 2,75 RM.

Kroger, Er as t. Erchnzungen- zum Burgerllehen Gesetzbueb mit Anmerkungen und Sachregister:

1. Band: Allgemeiner Teil, Recht der Schuldverhåltnisse, Sachenrecht, Familienrecht, Erbrecht. Mit Nachtrag. XLVIII, 803 und 38 Seiten. Preis in Ganzleinen geb. 15,75 RM.

2. Band: Einführungsgesetz zum Burgerlichen Gesetzbuch, PreuBisches Ausfohrungsgesetz zum Borgerlichen Geset2buch, PreuBische Verordnung zur Ausfahrung des Bargerlichen Gesetzbuchs. XXVII und 583 Seiten. Preis in Ganzleinen geb. 11,70 RM.

$\mathrm{LaB}, \mathrm{H} \mathrm{el} \mathrm{mut}$. Umwandlungsgesetz, Steuererleichterungsgesetz, Anleihestockgesetz. 240 Seiten. Preis in Ganzleinen geb. 5,- RM.

M a l le r, Fritz. StraBenverkehrareeht (Reichsgesetz Ober den Verkehr mit Kraftfahrzeugen und Reichsstraßenverkehrsordnung). Zehnte Auflage. Preis in Ganzleinen gebunden 22,50 RM.

Thieme, Paul. Grundbuchordnung für das Deutsche Reich in der Fassung vom 5. August 1935. 2. Auflage. 500 Seiten. Preis in Ganzleinen geb. $14,50 \mathrm{RM}$.

Wagemann, Gustav, und Marwitz, W. Dle PreuBisehe Pachtchutzordnung in der Fassung vom 19. 9. 27. 4. Auflage. VIII und 380 Seiten. Preis in Ganzleinen geb. 10,80 RM.

Winckler. Ernst. Gesets Gber Depot- and Depositengesehalte ram 26. 6. 25. 48 Seiten. Preis geh. 1,35 RM.

\section{Erbhofrecht.}

Z i m m er. Die Rechtsgescharte des Erbhofrechts. 207 Seiten. Prois in Ganzleinen geb. 4,50 RM. 


\section{Arbeitsrecht und Sozialrecht.}

K n a a k, R i c h a r d. Das Sehwerbesehsdigtengeset. XVI und 217 Seiten. Preis in Ganzleinen geb. 4,50 RM.

von Kunitzki-Ne u, Hans. Mutterschutz. 160 Seiten. Preis in Ganzleinen geb. 3,60 RM.

W a ge man n, G usta v. Die Arbeltsgesetze in einem Band. Mit einem Geleitwort von Reichsminister a. D. Dr. Brauns. Zweite Auflage. XV und 1176 Seiten. Preis in Ganzleinen geb. 10,- $\mathbf{R M}$.

Wagemann, Gustav, und $\mathrm{Cz}_{\mathrm{a}} \mathrm{lb}$ e. Die arbeltarechtllehen Vorschriften der allgemeinen Gesetze (Arbelitsrertragareeht). Preis in Ganzleinen geb. $15,-\mathrm{RM}$.

Wa geman $n$, Gustav, und Preiser, Fried ri c h. Franensehuts, Schutz der Jugendlichen und Kinder, Hausarbeitsgesets. 191 Seiten. Preis kart. 3,20 RM.

\section{Geistig-gewerblicher Rechtsschutz.}

Busse, R u d o l f. Patentgesetz vom 5. 5. 1936 nebst Gebrauchsmustergesetz vom 5. 5. 1936. Etwa 500 Seiten. Preis in Ganzleinen geb, etwa $13,50 \mathrm{RM}$.

Busse, R u d o lit. Warenzeichengesetz. Etwa 450 Seiten. Preis in Ganzleinen geb. etwa 12,- RM.

Pinzger, Werner. Das Deutsehe Gesebmackmusterreeht. 225 Seiten. Preis in Ganzleinen geb. 7,- RM.

\section{Zivilprozeßrecht und freiw. Gerichtsbarkeit.}

E is o ld, Hein rich. Verglelehsordnung. Vierte Bearbeitung in Vorbercitung. Preis in Ganzleinen geb. ca. 8,- RM.

J o n a s, M a r t i n. Das deutsehe Geriehtskostengesets. Mit Erlăuterungen und Kostentafeln sowie den Gebahrenordnungen. Zweite neubearbeitete und erweiterte Auflage. 464 Seiten. Preis in Ganzleinen geb. 9,- RM.

Rusche, Gerhard. Kostenordnung. 300 Seiten. Preis in Ganzleinen geb. 10,- RM.

$S$ c b a f e r, P a u l. Dle Einzelglaubigeranfechtung auf der Grundlage des Reichsgesetzes betr. die Anfechtung von Rechtshandlungen eines Schuldners auBerhalb eines Konkursveriahrens vom 21. 7. 1879. 263 Seiten. Preis in Ganzleinen geb. 6,75 RM. 
Zivilproze8ordnung for das Deutsche Reich (Fassung der Bekanntmachung von 8. 11. 33). Textausgabe mit lnhaltsverzeichnis und Sachregister 318 Seiten. Preis geb. 2,50 RM.

\section{Strafrecht.}

Fubse, Wilbe $1 \mathrm{~m}$. Milttarstralgesetzbuch. Zweite Auflage. $\mathrm{XV}$ und 338 Seiten. Preis in Ganzleinen geb. 7,20 RM.

H e 11 i g, A 1 b e r t. Jugendgerlchtggeselz. 368 Seiten. Preis geh. 5,40 RM.

Hoche, Werner, und $S \mathrm{ch}$ on er, Kurt. Gegetz gegen WaffenmiBbrauch. 55 Seiten. Preis kart. 1,45 RM.

W a g e man n, Gusta $\mathrm{v}$, und $\mathrm{K}$ ra n o ld. Dan Feld- und Forstpollzelpesetz i. d. F. vom 21. 1. 26, Das Forsldiebstahlogesetz. VIII und 273 Seiten. Preis in Ganzleinen geb. 9,- RM.

\section{Staatsrecht und allgemeine Staatslehre.}

Anschatz, Gerhard. Dle Verlassung des Deutsehen Reiebes vom 11. 8. 19. Vierte Bearbeitung. 14. Auflase. XXXXVIII und 800 Seiten. Preis in Ganzleinen geb. 10,- RM.

$\mathrm{S} \mathrm{c}$ b $\mathrm{atz}$ e l, W a l te $\mathrm{r}$. Das Deutsehe Stantangeh brigkeltareeht. 191 Selten. Preis in Ganzleinen geb. 4,50 RM.

S c b t z e l, W a l ter. Die Regelung der Staatsangehorighelt nach dem Wellkrleg. 391 Seiten. Preis in Ganzleinen geb. 7,20 RM.

Schlot tmann. Rudo If. Dle Verfassungen Englands, Nordamerikas, Frankreichs, der Schweiz und Deutsehlands in objektiver Darstellung, deutschen Verfassungstexten und ausląndischer Kritik. XV und 314 Seiten. Preis in Ganzleinen geb. 9,- RM.

Wa l d e cker, L u d wig. Die Verfassune des Frelatagtes Preuben vom 90. 11. 20. Zweite Auflage. 295 Seiten. Preis in Ganzleinen geb. 7.20 RM.

\section{Verwaltungsrecht.}

A rendts, Car l. Das Relehsversorqungsgesetz, Altrentnergesetz und Kriegapersonensehädengesetz. 593 Seiten. Preis in Ganzleinen geb. 13,50 RM.

A rendts, Carl. Die Versorgung der ebemaligen aktiven Olfiziere (einseblieBileb threr Hinterbliebenen) der alten Wehrmacht (Heer und Marine). Mit Nachtrag. XII, 500 und 114 Seiten. Preis in Ganzleinen geb. 14,30 RM. 


\section{Stiltes Redptobibliothet Nir. 105}

Die Befeşe des Dentiden Reids und ber beutjめen Ränder

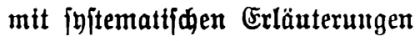

\section{Die \\ Cinzelgläubigeranfedhtung}

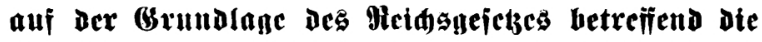
Mnfedtung non Biedtshnudunnen cines Edulaners nukgerhalb Des Sonfursucrfahrens vom 21 .3uli 1899, ill Der Fafiung Der Befanntmađung Des Pteidsfanzlex vom 20. Mlat 1898

unter $\mathfrak{B e r u ̈ u ̛ f i d u t i g u n g ~}$

Der Abänberung Durd bie Bergleidjaroronung vom 5. Juli 1927

exläuterı pou

\section{Dr. Daul Shaefer,}

Imtsgeridts

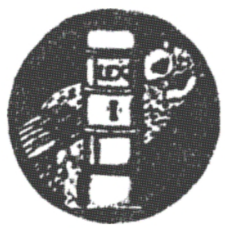

1930

$\mathfrak{B}$ erlag yon 

Wlle Redte vorbehalten.

(Bebntat bet 
